\title{
Alignment dependence of high-order harmonic generation from $\mathrm{CO}_{2}$
}

\author{
ANH-THU LE*†, X. M. TONG† and C. D. LIN† \\ $\uparrow$ Department of Physics, Cardwell Hall, Kansas State University, \\ Manhattan, KS 66506, USA \\ Institute of Materials Science, Graduate School of Pure and \\ Applied Science and Center for Computational Sciences, \\ University of Tsukuba, 1-1-1 Tennodai, Tsukuba, \\ Ibaraki 305-8573, Japan
}

(Received 15 July 2006; in final form 5 October 2006)

\begin{abstract}
High-order harmonic generation ( $\mathrm{HHG}$ ) from aligned $\mathrm{CO}_{2}$ is studied within the framework of the strong-field approximation (SFA). Our results are in qualitative agreements with recent pump-probe experiments. The experimentally observed inverted modulation in $\mathrm{HHG}$ signals as a function of pump-probe delay time has previously been attributed to the quantum interference from the two oxygen centres. Our results, however, indicate that this is not necessary for the inverted modulation. The angular dependence of the HHG and the evolution of the HHG yield as functions of delay time are influenced strongly by the depletion of the ground state and, therefore, are sensitive to the probe laser intensity.
\end{abstract}

\section{Introduction}

High-order harmonic generation (HHG) from atoms is well studied and understood as a three-step process [1-3]. However, random alignment of sample molecules has prevented researchers from studying in detail the HHG from molecules until recently. The advancements in the molecular alignment and orientation techniques open a variety of new and exciting applications. In order to observe HHG from aligned molecules, the so-called pump-probe scheme has been widely used. In this scheme, the molecular alignment is achieved by exposing molecules to a short, relatively weak laser pulse (the pump) to create a rotational wave packet. This wave packet rephases after the pulse is over and the molecules are strongly aligned periodically at intervals separated by their fundamental rotational period [4]. To observe the alignment dependence of HHG, a second short laser pulse (the probe) is then used to generate HHG at different short intervals when the molecules undergo rapid change in their alignment.

On the theoretical side, an interesting interference effect has been predicted first from the calculated $\mathrm{HHG}$ spectra of a model $2 \mathrm{D} \mathrm{H}_{2}^{+}$by solving numerically the

\footnotetext{
*Corresponding author. Email: atle@phys.ksu.edu
} 
time-dependent Schrödinger equation (TDSE), assuming that the nuclei are fixed in space, by Lein et al. [5]. They found pronounced minima in the HHG power spectra which they later interpreted as the interference of the emissions from the two atomic centres, when the wavelength of the returning electron matches the typical distance between the nuclei. Similar interference has been found later in the realistic 3D model of $\mathrm{H}_{2}^{+}$by Lein et al. [6] and Kamta and Bandrauk [7] under the same fixed-nuclei approximation. Telnov and $\mathrm{Chu}[8]$ have also calculated the $\mathrm{HHG}$ for $\mathrm{H}_{2}^{+}$aligned along the laser polarization direction, but no signature of interference was reported.

In a recent paper, Kanai et al. [9] performed pump-probe experiments on $\mathrm{N}_{2}, \mathrm{O}_{2}$ and $\mathrm{CO}_{2}$ using an $800 \mathrm{~nm}$ laser. By comparing the 23rd harmonic signals versus the ionization yields as a function of the time delay between the two laser pulses (same polarization direction), they found that the two curves follow each other for $\mathrm{N}_{2}$ and $\mathrm{O}_{2}$, i.e. when the ionization yield is maximum (minimum) the HHG yield is also maximum (minimum). Noting that for $\mathrm{N}_{2}$ the ionization is maximal along the molecular axis [10], these results are in fact in good agreement with the measurements by Itatani et al. [11]. These results imply that the HHG yield and the ionization rate for each molecule follow the same alignment dependence and the recombination step in the HHG mechanism does not introduce additional alignment dependence. In contrast, they observed inverted modulation in the HHG signal for the 23rd harmonic from $\mathrm{CO}_{2}$ as a function of pump-probe delay time. That is, they found minima in $\mathrm{HHG}$ emission at maximum ionizations and vice versa. Most recently, Vozzi et al. [12] also confirmed the inverted modulation from $\mathrm{CO}_{2}$, but for the 33rd harmonic. Both groups attributed the origin of the inversion to the interference of the photon emission from the two oxygen centres in $\mathrm{CO}_{2}$ which are separated by about twice the distance between the two nuclei in $\mathrm{O}_{2}$ where no inversion was found.

Can one indeed conclude that the inverted modulation in the HHG versus the ionization spectra in $\mathrm{CO}_{2}$ as a manifestation of the interference in the recombination process? Note that the harmonic order for the interference minimum depends on the angle between the molecular axis and the laser polarization direction. In both experiments the molecules are only partially aligned by the pump pulse, thus the measured HHG signals should be averaged over the angular distributions of the molecules, and it is not clear that the minimum predicted from the interference model in fixed nuclei approximation can survive after the average. Furthermore, if the interference model is indeed the correct interpretation, then it should occur at the same harmonic order, independent of the laser intensity of the probe pulse. In the experiments by Kanai et al. and Vozzi et al., the inversion was found at different harmonic orders.

In this paper, we extend our previous work [13] and show that there are other possible interpretations for the observed inverted modulation in $\mathrm{CO}_{2}$. In order to compare with experiments, in our analysis the anisotropic angular distributions of the molecules at each time delay after the pump pulse are taken into account in obtaining the calculated HHG spectra and the ionization yields. To calculate the HHG spectra for a fixed alignment, we used the Lewenstein model [3] for molecules as extended in Zhou et al. [14]. This model has been shown [14] to be able to interpret the experimental results reported so far for $\mathrm{N}_{2}$ and $\mathrm{O}_{2}[9,11,15]$. 
The rest of this paper is organized as follows. In section 2 we discuss the alignment of the molecules in the ultrashort laser field. Section 3 presents results from the molecular tunnelling (MO-ADK) theory applied to $\mathrm{CO}_{2}$. We show that the ionization signals are in phase with the molecular alignment. The main results on HHG are presented in section 4. In section 5 we discuss different formulations of the Lewenstein model and compare the results from the length and velocity formulations. The last section gives a brief summary and conclusions.

\section{Alignment of molecules by lasers}

When a molecule is placed in a short laser field (the pump), the laser will excite a rotational wave packet (coherent superposition of rotational states) in the molecule. By treating $\mathrm{CO}_{2}$ as a rigid rotor [16, 17], the rotational motion of the molecule with initial state $\Psi(\theta, \phi, t=-\infty)=|J M\rangle$ evolves in the laser field following the time-dependent Schrödinger equation (atomic units $m=e=\hbar=1$ are used throughout the paper, unless otherwise indicated)

$$
\mathrm{i} \frac{\partial \Psi(\theta, \phi, t)}{\partial t}=\left[B \mathbf{J}^{2}-\frac{E(t)^{2}}{2}\left(\alpha_{\|} \cos ^{2} \theta+\alpha_{\perp} \sin ^{2} \theta\right)\right] \Psi(\theta, \phi, t) .
$$

Here $B$ is the rotational constant, $\alpha_{\|}$and $\alpha_{\perp}$ are the anisotropic polarizabilities in parallel and perpendicular directions with respect to the molecular axis, respectively. The above equation with $B=0.39 \mathrm{~cm}^{-1}, \alpha_{\|}=4.05 \AA^{3}$ and $\alpha_{\perp}=1.95 \AA^{3}$ [18] is then solved for each initial rotational state $|J M\rangle$ using the split-operator method [19]. In our calculation the pump laser $E(t)$ for aligning the molecules is assumed to be a Gaussian pulse, with duration of $50 \mathrm{fs}$ (full width at half maximum (FWHM)) and peak intensity of $3 \times 10^{13} \mathrm{~W} \mathrm{~cm}^{-2}$, and a mean wavelength of $800 \mathrm{~nm}$. We also assume the Boltzmann distribution of the rotational levels at the initial time. The rotational temperature of $\mathrm{CO}_{2}$ molecules is taken to be $40 \mathrm{~K}$. With these assumptions, the time-dependent alignment distribution can be obtained as

$$
\rho(\theta, t)=\sum_{J M} \omega_{J M}|\Psi(\theta, \phi, t)|^{2}
$$

where $\omega_{J M}$ is the weight according to the Boltzmann distribution. For the system under consideration, the angular distribution or the alignment does not depend on the azimuth at angle $\phi$. The two equations above allow determination of the time evolution of the alignment distribution of the molecules in the laser field, as well as the rotational revivals after the laser has been turned off. Note that we assume that the aligning laser is weak enough so the molecules remain in the ground state and no ionization occurs.

\section{Molecular tunnelling ionization theory}

In the experiments by Kanai et al. [9] the measurements were carried out simultaneously for both ionization and HHG signals. In this section, we use the molecular 
tunnelling ionization (MO-ADK) theory [20] to describe the ionization process. In this theory, the ionization rate of a molecule in a static electric field $F$ is given by

$$
w_{\text {stat }}(F, \mathbf{R})=\sum_{m^{\prime}} \frac{B^{2}\left(m^{\prime}\right)}{2^{\left|m^{\prime}\right|}\left|m^{\prime}\right| !} \frac{1}{\kappa^{2 Z_{\mathrm{c}} / \kappa-1}}\left(\frac{2 \kappa^{3}}{F}\right)^{2 Z_{\mathrm{c}} / \kappa-\left|m^{\prime}\right|-1} \exp \left(-2 \kappa^{3} / 3 F\right),
$$

with $Z_{\mathrm{c}}$ being the effective Coulomb charge, $\kappa=\left(2 I_{\mathrm{p}}\right)^{1 / 2}$ and $I_{\mathrm{p}}$ being the ionization potential. Here $B\left(m^{\prime}\right)$ is given by

$$
B\left(m^{\prime}\right)=\sum_{l} C_{l} D_{m^{\prime}, m}^{l}(\mathbf{R})(-1)^{m^{\prime}}\left(\frac{(2 l+1)\left(l+\left|m^{\prime}\right|\right) !}{2\left(l-\left|m^{\prime}\right|\right)}\right)^{1 / 2},
$$

with $D_{m^{\prime}, m}^{l}(\mathbf{R})$ being the rotation matrix and $\mathbf{R}$ the Euler angles between the molecular axis and the field direction. The parameters $C_{l}$ are determined from the valence electron wavefunction of the molecule in the asymptotic region. We calculated these coefficients using the multiple-scattering method [21] and within the Hartree-Fock approximation using the GAMESS code [22]. The results for $C_{l}$ from the multiple-scattering method (or GAMESS) are 2.88 (2.94), 1.71 (1.24) and $0.427(0.317)$ for $l=2,4$ and 6 , respectively. The two sets of coefficients are quite close so in the following we only use the multiple-scattering results.

The ionization rate in a low frequency laser field is obtained by averaging over a laser cycle and is given by

$$
w(F, \mathbf{R})=\left(\frac{3 F}{\pi \kappa^{3}}\right)^{1 / 2} w_{\text {stat }}(F, \mathbf{R})
$$

where $F$ now stands for the peak field strength.

In figure $1(a)$ we show the alignment-dependent ionization rate for $\mathrm{CO}_{2}$ molecules at laser intensities of $1 \times 10^{13}, 5 \times 10^{13}, 1 \times 10^{14}$ and $2 \times 10^{14} \mathrm{~W} \mathrm{~cm}^{-2}$. The rates have been normalized to that of $\theta=0$. We can see that the ionization rate peaks at $\theta$ near $25^{\circ}$. With higher laser intensities the dependence of the ionization rate on $\theta$ becomes weaker. Note that, for the parallel alignment, although the highest occupied molecular orbital (HOMO) of $\mathrm{CO}_{2}$ has a nodal line along the laser polarization direction, the ionization rate does not vanish. This is especially true for high intensities. In this respect, we also note that at higher intensities, $\mathrm{CO}_{2}$ ionization rate behaves somewhat closer to that of $\mathrm{N}_{2}$, which also peaks near $\theta=0$.

The ionization rate as a function of pump-probe delay time is plotted in figure $1(b)$. The calculations were performed with the pump laser parameters as described in the previous section. The probe laser with intensity of $2 \times 10^{14}$ is polarized in parallel to the pump laser polarization. Here the ionization signals have been normalized to that of isotropic molecular distribution. Clearly, the ionization signals are in phase with the degree of alignment $\left\langle\cos ^{2} \theta\right\rangle$, shown as the dashed curve. This is in agreements with the results by Kanai et al. [9] and Vozzi et al. [12]. 

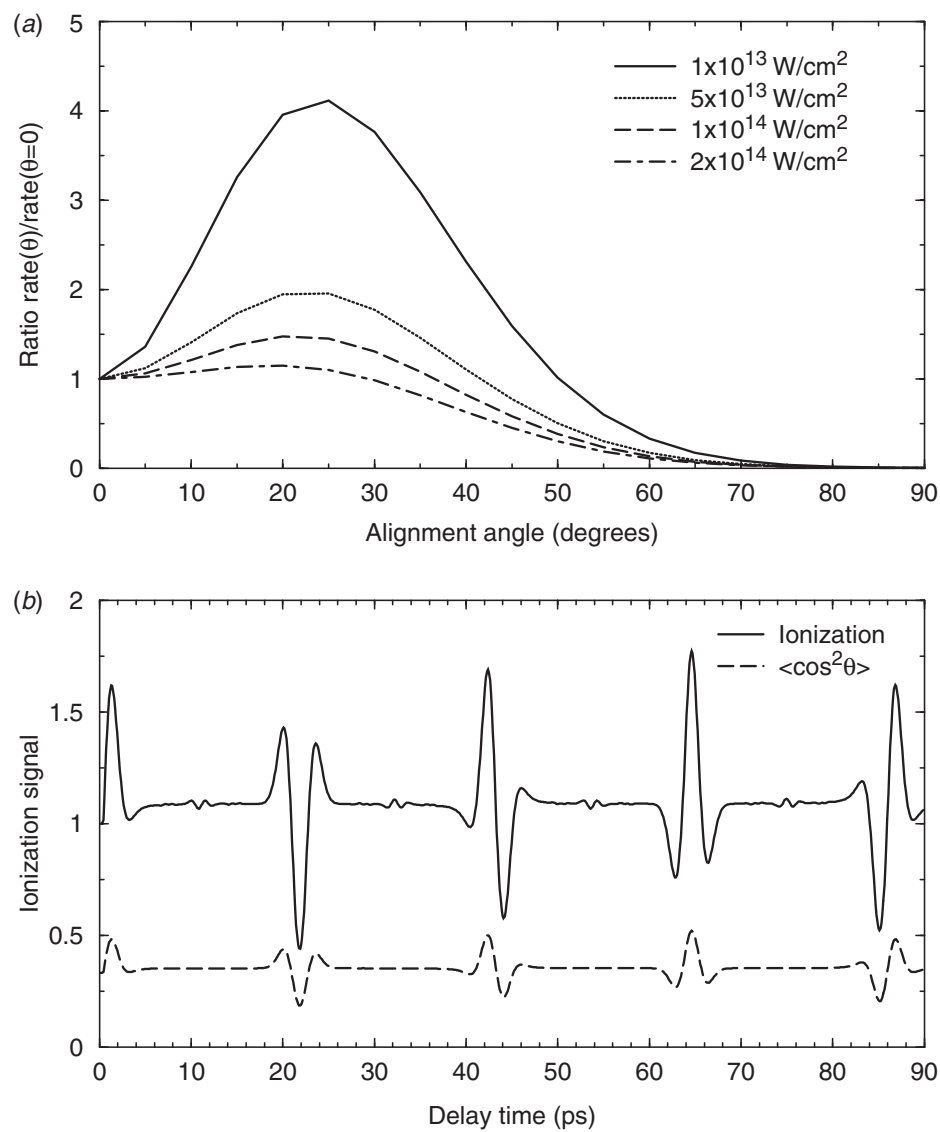

Figure 1. (a) Alignment dependence of ionization rate for $\mathrm{CO}_{2}$ molecules at laser intensities shown on labels. (b) Ionization rate as a function of pump-probe delay time for the probe intensity of $2 \times 10^{14}$. Also shown is $\left\langle\cos ^{2} \theta\right\rangle$, which conveniently measures the degree of alignment. The pump laser parameters are given in section 2 .

\section{High-order harmonic generation: Lewenstein model in the length form formulation}

In this section, we use the Lewenstein model, as extended by Zhou et al. [14] to calculate the HHG spectra for a molecule with fixed alignments in a probe laser pulse. The induced dipole moment of a molecule aligned along the $z$ axis in a laser field $E(t)$, linearly polarized on the $y-z$ plane with an angle $\theta$ with respect to the molecular axis can be written in the form

$$
\begin{aligned}
D(t)= & \mathrm{i} \int_{0}^{\infty} \mathrm{d} \tau\left(\frac{\pi}{\epsilon+\mathrm{i} \tau / 2}\right)^{3 / 2}\left[\sin \theta d_{y}^{*}(t)+\cos \theta d_{z}^{*}(t)\right] \\
& \times\left[\sin \theta d_{y}(t-\tau)+\cos \theta d_{z}(t-\tau)\right] E(t-\tau) \\
& \times \exp \left[-\mathrm{i} S_{\mathrm{st}}(t, \tau)\right] a^{*}(t) a(t-\tau)+c . c .
\end{aligned}
$$


where $\mathbf{d}(t) \equiv \mathbf{d}\left[\mathbf{p}_{\mathrm{st}}(t, \tau)-\mathbf{A}(t)\right], \mathbf{d}(t-\tau) \equiv \mathbf{d}\left[\mathbf{p}_{\mathrm{st}}(t, \tau)-\mathbf{A}(t-\tau)\right]$ are the transition dipole moments between the ground state and the continuum state, and $\mathbf{p}_{\text {st }}(t, \tau)=\int_{t-\tau}^{t} \mathbf{A}\left(t^{\prime}\right) \mathrm{d} t^{\prime} / \tau$ is the canonical momentum at the stationary points, with A the vector potential. The quasiclassical action at the stationary points for the electron propagating in the laser field is

$$
S_{\mathrm{st}}(t, \tau)=\int_{t-\tau}^{t}\left(\frac{\left[\mathbf{p}_{\mathrm{st}}(t, \tau)-\mathbf{A}\left(t^{\prime}\right)\right]^{2}}{2}+I_{\mathrm{p}}\right) \mathrm{d} t^{\prime},
$$

where $I_{\mathrm{p}}$ is the ionization potential of the molecule. In equation (6), $a(t)$ is introduced to account for the ground state depletion.

In figure 2, we plot the $\mathrm{HHG}$ power spectra calculated within our extended Lewenstein model from $\mathrm{CO}_{2}$ molecules aligned at three fixed angles $\theta=30^{\circ}, 50^{\circ}$

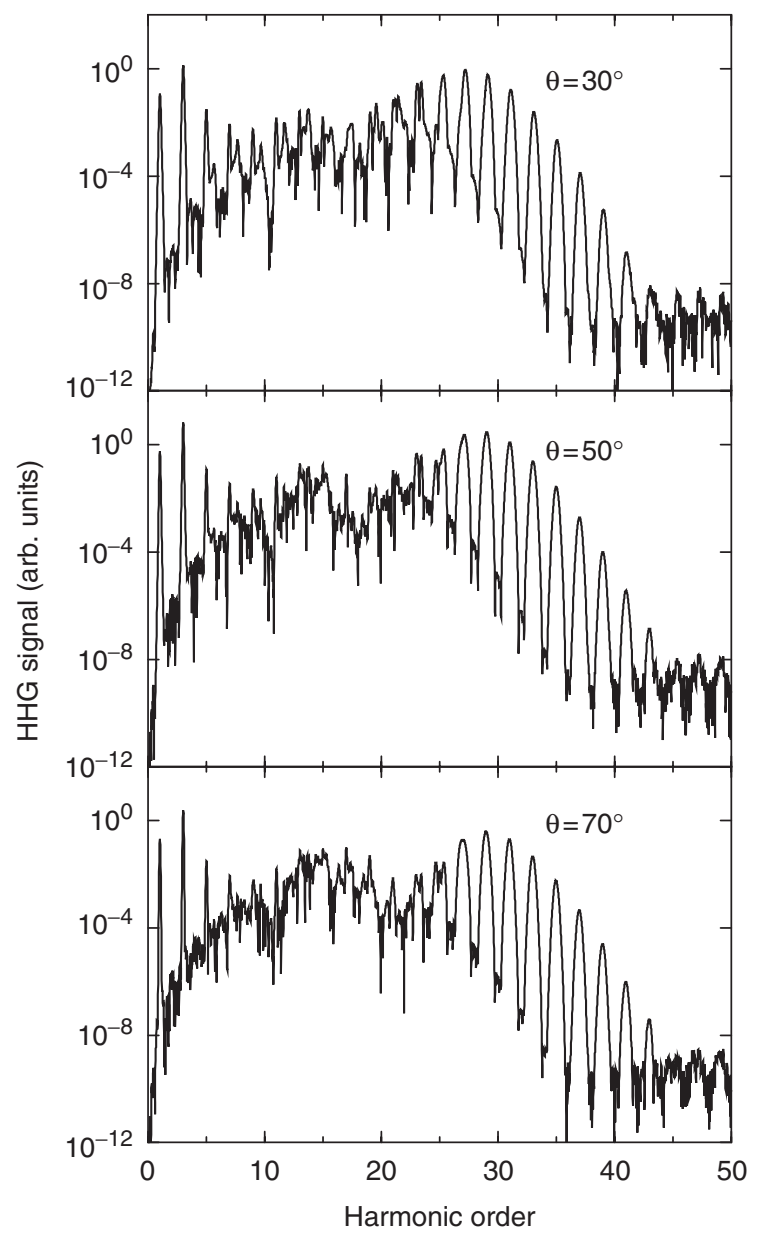

Figure 2. HHG power spectra for selective fixed alignment angles $\theta=30^{\circ}$ (top), $50^{\circ}$ (middle) and $70^{\circ}$ (bottom) at laser intensity of $1.5 \times 10^{14} \mathrm{~W} \mathrm{~cm}^{-2}$. For other laser parameters, see text. 
and $70^{\circ}$. The ground state electronic wavefunction of $\mathrm{CO}_{2}$ was obtained from the GAMESS code [22]. In order to account for the depletion of the ground state, we approximate the ground state amplitude by $a(t)=\exp \left[-\int_{-\infty}^{t} W\left(t^{\prime}\right) / 2 \mathrm{~d} t^{\prime}\right]$, with the ionization rate $W\left(t^{\prime}\right)$ obtained from the MO-ADK theory (see, the previous section). For this calculation, we took a Gaussian pulse of $30 \mathrm{fs}$ duration, peak intensity of $1.5 \times 10^{14} \mathrm{~W} \mathrm{~cm}^{-2}$, and a mean wavelength of $800 \mathrm{~nm}$. We note from these figures that there is no obvious 'interference minimum' for the harmonics above about $N=25$ for all the 31 alignment angles between $0^{\circ}$ and $90^{\circ}$ that we have calculated.

Based on these results does it mean that one should not expect inverted modulation in the pump-probe experiments? Figure 3 shows the HHG signal as a function of delay time for the 17th (left, top panel), the 23rd (left, middle) and the $33 \mathrm{rd}$ harmonics (left, bottom) at a probe laser intensity of $2 \times 10^{14} \mathrm{~W} \mathrm{~cm}^{-2}$. The signals have been normalized to that of the isotropic molecular distribution. Here the pump and probe pulses are polarized parallel. For reference, we also plot here the evolution of $\left\langle\cos ^{2} \theta\right\rangle$ (dashed curves), as it is a measure of the degree of alignment.
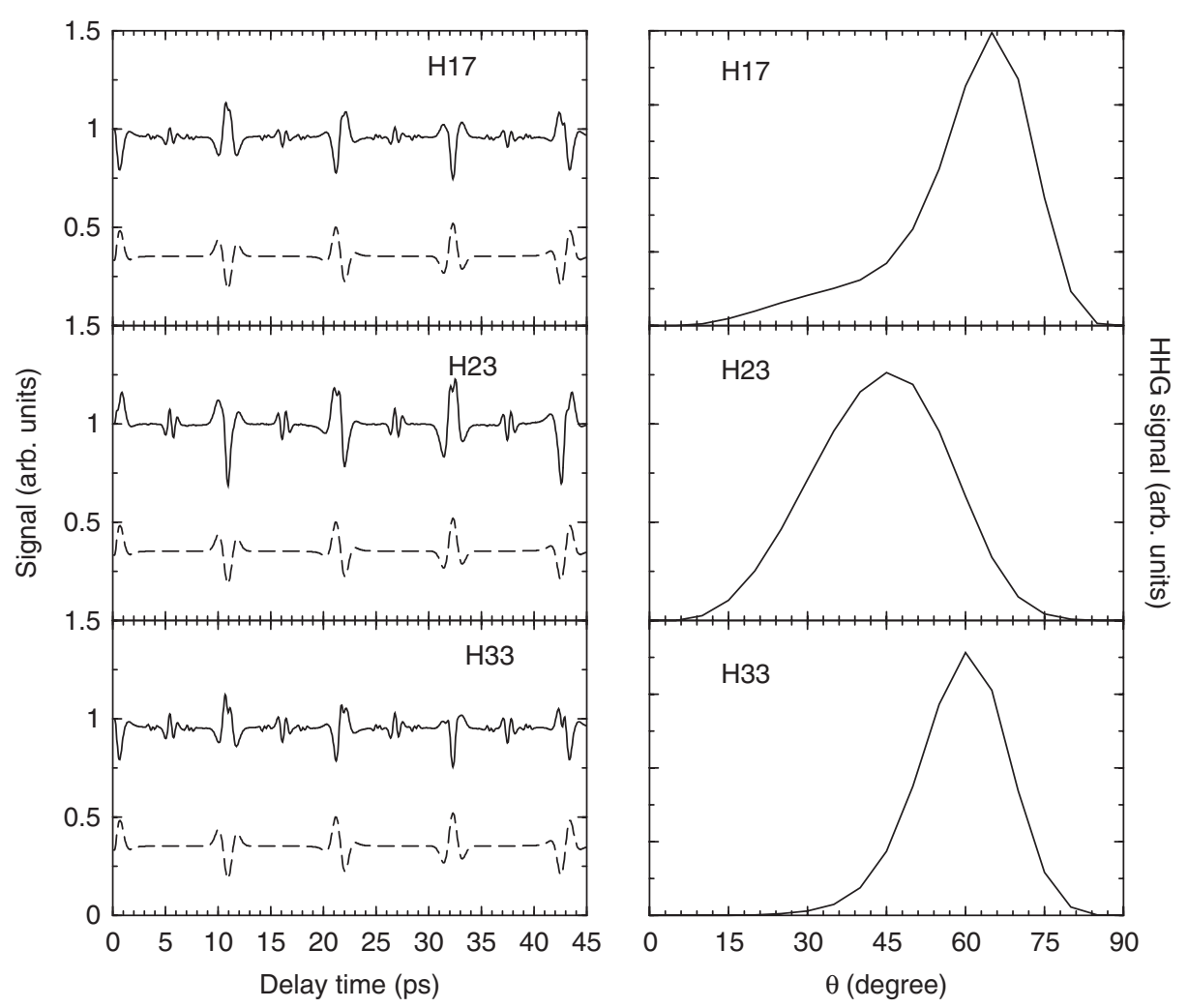

Figure 3. Time evolution of the selective harmonics (left column) and their alignment dependence (right column) at the probe laser intensity of $2 \times 10^{14} \mathrm{~W} \mathrm{~cm}^{-2}$. For other laser parameters, see text. Dashed curves correspond to the evolution of $\left\langle\cos ^{2} \theta\right\rangle$. 
Note that the ionization signals follow closely the evolution of $\left\langle\cos ^{2} \theta\right\rangle$ (see figure $1(b)$ ). One can see clearly the inverted modulation for the 17 th and 33rd harmonics. This is in qualitative agreement with the results by Kanai et al. [9]. The right panels show the alignment dependence of these harmonics. We notice that for the 17 th and 33rd harmonics, the distribution is peaked at angles greater than about $50^{\circ}$, whereas it is peaked at about $45^{\circ}$ for the $23 \mathrm{rd}$ harmonic. We will come back to this point later. Note that the calculations by de Nalda et al. [23] indicate that the alignment dependence of $\mathrm{HHG}$ from $\mathrm{CO}_{2}$ for lower orders $(N=9-17)$ have peaks at angles greater than $45^{\circ}$ too.

Let us try to understand these results. In figure 4 we show the close-up of the time evolution for the HHG near half-revival $(t=21.3 \mathrm{ps})$. We also plot in the upper panel the ionization yield (dotted curve), normalized to that of the isotropic distribution.

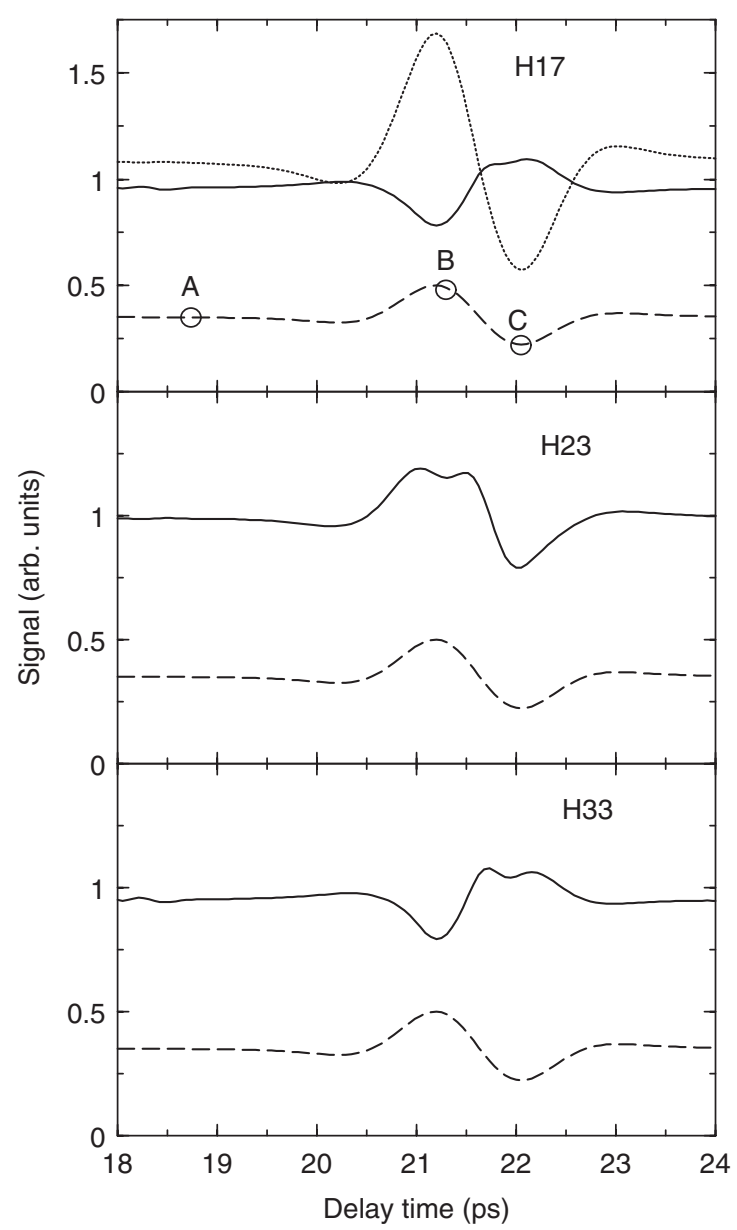

Figure 4. Same as figure 3 but near half-revival. On the top panel the ionization signal (dotted curve) is also shown. 
Recall that the time evolution of the HHG yield for a particular harmonic can be written in the form

$$
g_{2 n+1}(t)=2 \pi \int_{0}^{\pi} \rho(\theta, t) \bar{g}_{2 n+1}(\theta) \mathrm{d} \theta,
$$

where $\rho(\theta, t)$ is the weighted angular distribution (i.e. multiplied by $\sin \theta)$ of the molecules, $\bar{g}_{2 n+1}(\theta)$ is the HHG yield for the $(2 n+1)$ th harmonic from the molecules aligned at a fixed angle $\theta$ and $t$ is the delay time. Here we already account for the fact that the system has azimuthal symmetry. Figure 5 shows the polar plot of the weighted angular distribution (dashed curves) at three different pump-probe delays 18.7 (upper panels), 21.3 (middle panels), and $22.1 \mathrm{ps}$ (bottom panels), denoted respectively as A, B and C on figure 4. Also plotted are the HHG yields (solid curves)

(a)

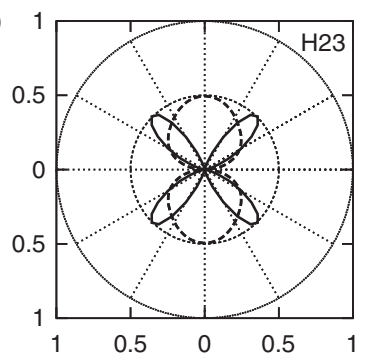

(b)

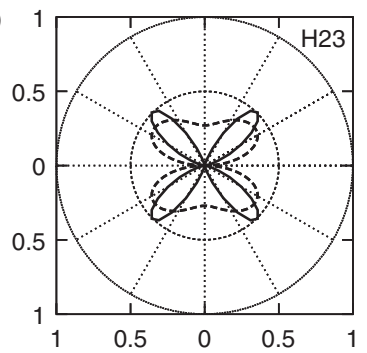

(c)

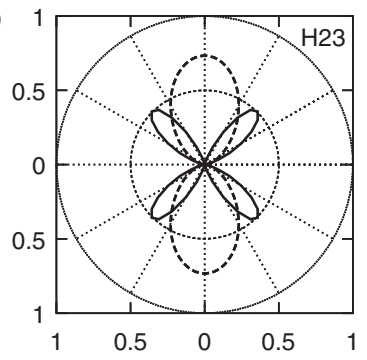

(d)

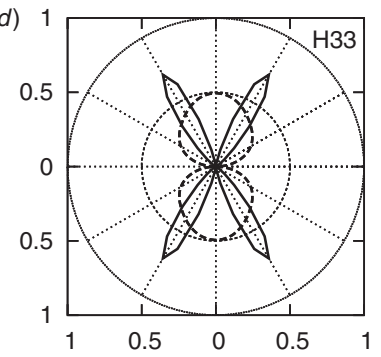

(e)

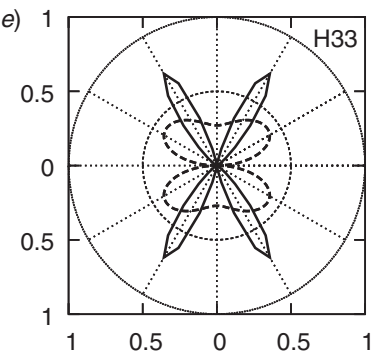

$(f)$

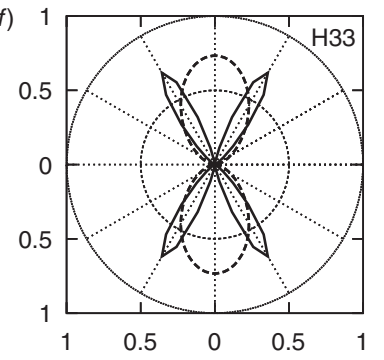

Figure 5. Polar plot of the weighted alignment distribution of the molecules (dashed curves) at different time delays $t=18.7 \mathrm{ps}$ (top), $21.3 \mathrm{ps}$ (middle) and $22.1 \mathrm{ps}$ (bottom) (see also figure 4). Also plotted is the alignment dependence (solid curves) for the $23 \mathrm{rd}$ harmonic (left column) and the 33rd harmonic (right column). The laser parameters are the same as for figure 3. Laser polarization is along the horizontal axes. 
for the 23rd harmonic (left column) and the 33rd harmonic (right column). Let us now consider the 23 rd harmonic. Due to the symmetry, we need only consider $\theta$ in the range $\left[0^{\circ}-90^{\circ}\right]$. First, we notice that the $\mathrm{HHG}$ yield is well localized in the angular range $\left[30^{\circ}-60^{\circ}\right]$, as can also be seen from figure 3 (right, middle panel). For that range of alignment, as can be seen from the figure, the weighted alignment distribution is largest for B and smallest for C. Therefore, according to equation (8), the time evolution of HHG is not inverted, which is consistent with the results on figure 3. In contrast, the 33rd harmonic yield is even narrower with the peak near $60^{\circ}$. For this small range of alignment, the weighted alignment distribution is smallest for $\mathrm{B}$ and largest for $\mathrm{C}$, which leads to the inverted modulation, according to equation (8).

At lower probe laser intensities, the depletion effect becomes weaker. As consequences, the HHG peaks are shifted to the smaller alignment angles. According to the discussion above, this would lead to a drastic change in the behaviour for the time evolution of $\mathrm{HHG}$ yield. In fact, at $1.5 \times 10^{14} \mathrm{~W} \mathrm{~cm}^{-2}$, the results still do not differ qualitatively from that of $2 \times 10^{14} \mathrm{~W} \mathrm{~cm}^{-2}$. For intensity of $1 \times 10^{14} \mathrm{~W} \mathrm{~cm}^{-2}$, we plot in figure 6 the $\mathrm{HHG}$ yields as functions of delay time for three harmonics of the 17th, 23rd and 33rd orders (left panels). Other laser
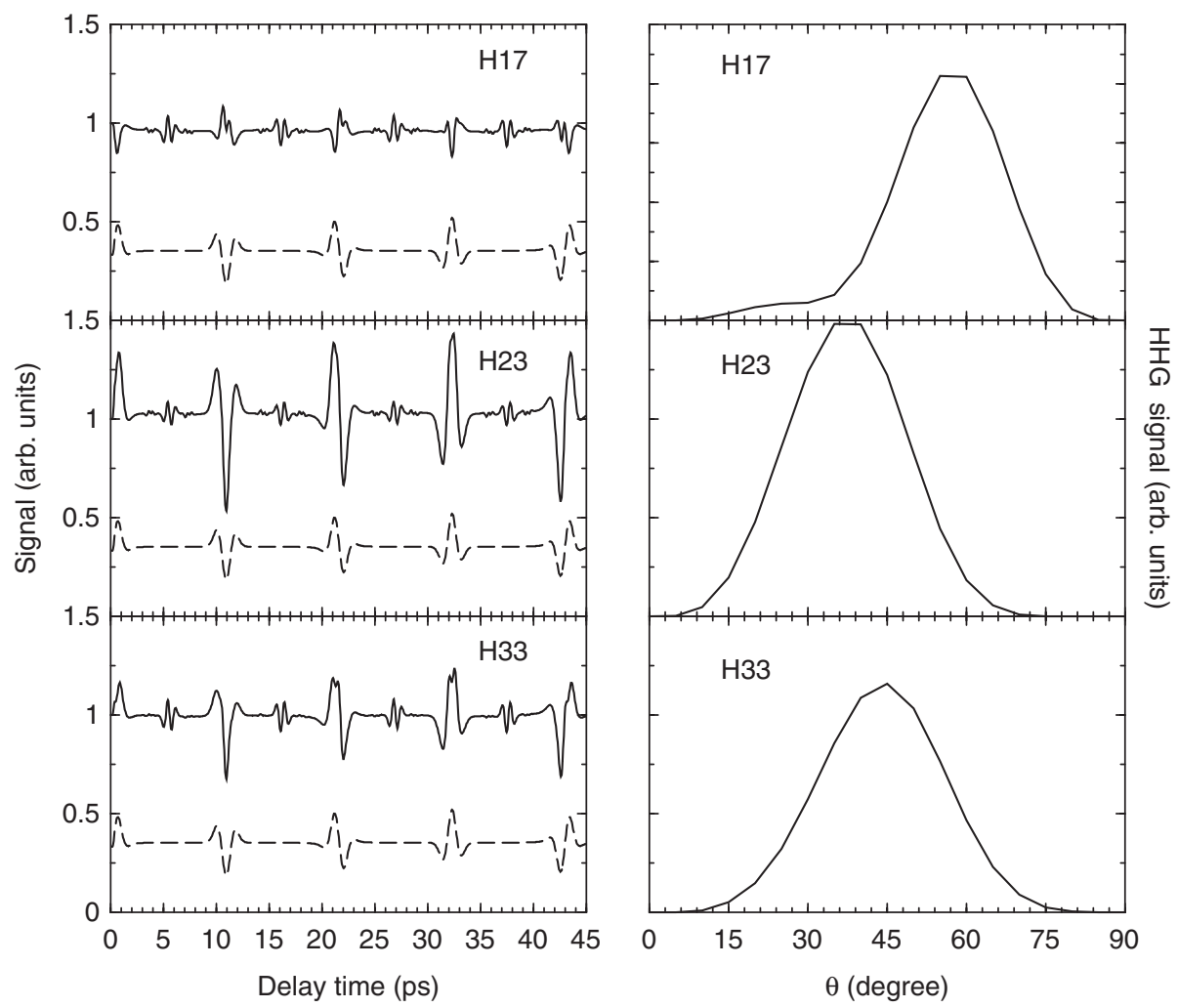

Figure 6. Same as figure 3 but for the probe laser intensity of $1 \times 10^{14} \mathrm{~W} \mathrm{~cm}^{-2}$. 
parameters are the same as for figure 3. As one can see, the HHG dependence on the alignment (right panels) is shifted towards smaller angles, as compared to that from figure 3 . As a consequence, the 33rd harmonic is not inverted. Thus one may suspect that the different harmonics where the inversion occurs in the experiment of Kanai et al. [9] and of Vozzi et al. [12] are due to the different probe intensities used. Future experiments with several probe laser intensities are therefore highly desirable in order to distinguish the present interpretation from the interference model.

\section{Calculations within the velocity form formulation}

In their original paper [3], Lewenstein et al. used the length (or dipole) form formulation. More specifically, the HHG power spectra are obtained from the Fourier components of the induced dipole moment $D(t)=\langle\Psi(t)|x| \Psi(t)\rangle$ as given by

$$
P(\omega) \propto|a(\omega)|^{2}=\left|\int \frac{\mathrm{d}^{2} D(t)}{\mathrm{d} t^{2}} \exp (\mathrm{i} \omega t) \mathrm{d} t\right|^{2} \approx \omega^{4}|D(\omega)|^{2} .
$$

The results presented in the previous section were carried out using this formulation. Some authors also use the so-called acceleration form (A-form), which utilizes the Ehrenfest theorem to directly calculate the dipole acceleration [5-7]

$$
|a(\omega)|^{2}=\mid \int\left\langle\Psi ( t ) \left|\mathbf { e } \cdot \left[\nabla V(r)+\left.\mathbf{E}(t)|\Psi(t)\rangle \exp (\mathrm{i} \omega t) \mathrm{d} t\right|^{2} .\right.\right.\right.
$$

It has been shown that at low intensities, the above two forms are almost identical [24], provided the exact wavefunctions are used. Finally there is another way, intermediate between the above two approaches, the so-called velocity form (V-form). Within this approach, instead of $D(t)$ one first calculates the dipole velocity

$$
V(t)=\langle\Psi(t)|\hat{p}| \Psi(t)\rangle
$$

and the HHG power spectra are then given by

$$
P(\omega) \propto|a(\omega)|^{2}=\left|\int \frac{\mathrm{d} V(t)}{\mathrm{d} t} \exp (\mathrm{i} \omega t) \mathrm{d} t\right|^{2} \approx \omega^{2}|V(\omega)|^{2} .
$$

Due to the approximations made in the Lewenstein model, the three formulations above can lead to somewhat different results. It is interesting to note that in the velocity formulation the positions of the interference minima agree quite well with the prediction of the simple two-emitter model by Lein et al. [6], if the ionization potential $I_{\mathrm{p}}$ is included in determination of the electron wavelength.

In figure 7 we show the HHG spectra obtained within the V-form calculations at laser intensity of $2 \times 10^{14} \mathrm{~W} \mathrm{~cm}^{-2}$ for the two alignment angles $\theta=15^{\circ}$ and $30^{\circ}$. The other laser parameters are the same as for figure 2. One can see the interference minima near the 33rd harmonic, as indicated by the arrows on the figure. In fact, this is where the two-emitter model predicts the interference minima to occur [12]. In these calculations we choose not to include the depletion of the ground state in order to have more pronounced interference effects (also see figure 8 below). 


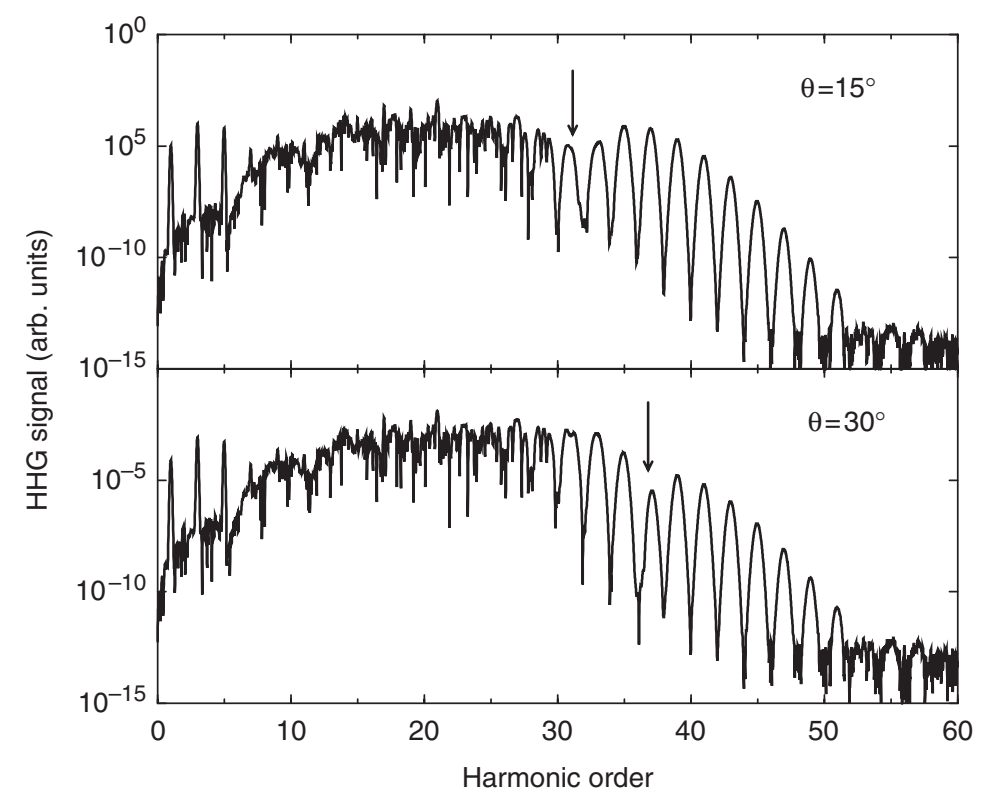

Figure 7. HHG power spectra, calculated with the V-form formulation, for fixed alignment angles $\theta=15^{\circ}$ (upper panel) and $30^{\circ}$ (lower panel) at laser intensity of $2 \times 10^{14} \mathrm{~W} \mathrm{~cm}^{-2}$. The arrows indicate the positions of the interference minima.

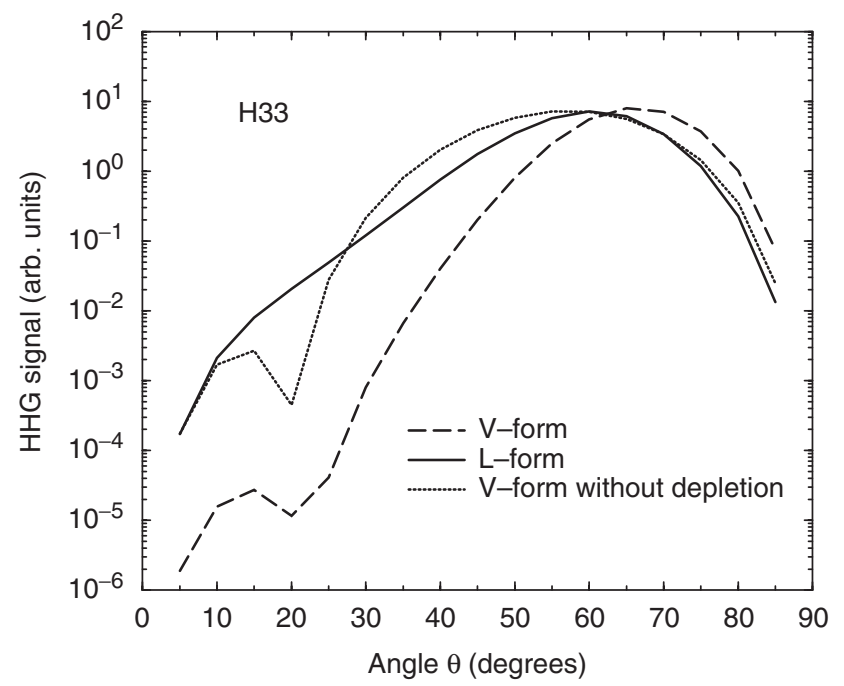

Figure 8. Alignment dependence of the 33rd harmonic at the laser intensity of $2 \times 10^{14} \mathrm{~W} \mathrm{~cm}^{-2}$. Note the $\log$ scale for the vertical axis. 
Do these interference minima have any significant effect on the measured HHG signal, as a function of pump-probe delay time? To answer this question, it is instructive to look at the alignment dependence of the generated harmonics. Figure 8 compares the alignment dependence for the 33rd harmonic, calculated within the velocity (dashed curve) and length forms (solid curve). The overall shape from the two calculations agrees quite well, with the V-form result shifted a bit to larger alignment angles $\theta$. Also shown in the figure is the results from V-form calculations without depletion of the ground state taken into account. One can see the interference minimum near $\theta=20^{\circ}$, which is present only in the $\mathrm{V}$-form result. However, the signals from these alignments are 4-5 orders of magnitude smaller compared to that from the peak at about $65^{\circ}$ (note the log scale). Therefore they do not contribute to the $\mathrm{HHG}$ signals, as one averages over the alignment distribution of the molecules.

We note, however, that within the V-form formulation, the calculated alignment dependence for all harmonics from the 11th order up to the 49th order is peaked at angles greater than $45^{\circ}$ even if no depletion is taken into account. In other words, one would expect the modulation inversion for most of the harmonics. This is different from the results within the length form formulation of the previous section.

\section{Conclusions}

In conclusion, we have shown that according to our model calculations the inverted modulations are not necessarily associated with the two-centre interference which has been used to explain the results of the recent experiments by Kanai et al. [9] and Vozzi et al. [12]. The angular distributions of the partially aligned molecules by the pump pulse should be taken into account in the interpretation of the experimental HHG data in pump-probe experiments. Our results also show that the angular dependence and the time evolution of the HHG yield are influenced strongly by the depletion of the ground state and are, therefore, sensitive to the probe laser intensity.

On the theoretical side, what is the evidence for two-centre interference of HHG from molecules that are fixed in space so far? The best evidence is for the case of $\mathrm{H}_{2}^{+}$by solving the TDSE with fixed alignments [5-7]. These calculations show that the HHG spectra exhibit minima and the positions of the minima agree with the simple two-emitter model. In these calculations, the HHG spectra were calculated only in the acceleration gauge. Calculations by Telnov and $\mathrm{Chu}$ [8], also for $\mathrm{H}_{2}^{+}$, where they showed results using the length, velocity and acceleration forms are converged, did not report any signature of the interference. Furthermore, within the simple two-emitter model, Lein et al. [6] were able to get the interference minima and maxima positions in agreement with their TDSE results for $\mathrm{H}_{2}^{+}$without including the ionization potential $I_{\mathrm{p}}$ in the determination of the electron wavelength. Using the Lewenstein model in the velocity form for $\mathrm{H}_{2}^{+}$and $\mathrm{CO}_{2}$, we have found minima in the HHG spectra and the positions of the minima are in agreement with the two-emitter model, but only with $I_{\mathrm{p}}$ included in the determination of the electron wavelength. Using the length form the predicted positions of interference minima occur at much larger harmonic orders, as compared to the available TDSE results. 
Thus, while there is evidence of interference minima in the HHG from molecules that are fixed in space, whether the positions of the minima are given by the simple twoemitter model is less certain. Additional model calculations using TDSE and with HHG spectra calculated with all three gauges would help to clarify this confusion. Finally, whether the predicted HHG minima for molecules fixed in space would appear in typical pump-probe experiments would require more careful analysis, after the partial alignment of the molecules from the pump pulse is accounted for. In addition, the intensity of the probe pulse should be limited so that no depletion of the molecules occurs to further complicate the interpretation of the experimental data.

\section{Acknowledgement}

This work was supported by the Chemical Sciences, Geosciences and Biosciences Division, Office of Basic Energy Sciences, Office of Science, US Department of Energy.

\section{References}

[1] K.C. Kulander, K.J. Schafer and J.L. Krause, in Super Intense Laser-Atom Physics, in the NATO Advanced Study Institute Series (Plenum Press, New York, 1993), p. 95.

[2] P.B. Corkum, Phys. Rev. Lett. 711994 (1993).

[3] M. Lewenstein, Ph. Balcou, M.Yu. Ivanov, et al., Phys. Rev. A 492117 (1994).

[4] H. Stapelfeldt and T. Seideman, Rev. Mod. Phys. 75543 (2003).

[5] M. Lein, N. Hay, R. Velotta, et al., Phys. Rev. Lett. 88183903 (2002).

[6] M. Lein, P.P. Corso, J.P. Marangos, et al., Phys. Rev. A 67023819 (2004).

[7] G.L. Kamta and A.D. Bandrauk, Phys. Rev. A 71053407 (2005).

[8] D.A. Telnov and S.-I. Chu, Phys. Rev. A 71013408 (2005).

[9] T. Kanai, S. Minemoto and H. Sakai, Nature 435470 (2005).

[10] I.V. Litvinyuk, K.F. Lee, P.W. Dooley, et al., Phys. Rev. Lett. 90233003 (2003).

[11] J. Itatani, D. Zeidler, J. Levesque, et al., Phys. Rev. Lett. 94123902 (2005).

[12] C. Vozzi, F. Calegari, E. Benedetti, et al., Phys. Rev. Lett. 95153902 (2005).

[13] A.T. Le, X.M. Tong and C.D. Lin, Phys. Rev. A 73 041402(R) (2006).

[14] X.X. Zhou, X.M. Tong, Z.X. Zhao, et al., Phys. Rev. A 71 061801(R) (2005); ibid. 72 $033412(2005)$.

[15] J. Itatani, J. Levesque, D. Zeidler, et al., Nature 432867 (2004).

[16] T. Seideman, J. Chem. Phys. 1037887 (1995).

[17] J. Ortigoso, M. Rodriguez, M. Gupta, et al., J. Chem. Phys. 1103870 (1999).

[18] J.O. Hirschfelder, C.F. Curtiss and R.B. Bird, Molecular Theory of Gases and Liquids (Wiley, New York, 1954).

[19] X.M. Tong and S.I. Chu, Chem. Phys. 217119 (1997).

[20] X.M. Tong, Z.X. Zhao and C.D. Lin, Phys. Rev. A 66033402 (2002).

[21] D. Dill and J.L. Dehmer, J. Chem. Phys. 61692 (1974).

[22] M.W. Schmidt, K.K. Baldridge, J.A. Boatz, et al., J. Comput. Chem. 141347 (1993).

[23] R. de Nalda, E. Heesel, M. Lein, et al., Phys. Rev. A 69 031804(R) (2004).

[24] K. Burnett, V.C. Reed, J. Cooper, et al., Phys. Rev. A 453347 (1992). 